\title{
Bright light treatment for prevention of perioperative delirium in elderly patients
}

\section{Toyoe Taguchi}

Department of Acute and Critical Care in Adult Nursing, School of Nursing Science, Meiji University of I ntegrative Medicine. Kyoto, Japan

Correspondence: Toyoe Taguchi. Address: Department of Acute and Critical Care in Adult Nursing, School of Nursing Science, Meiji University of Integrative Medicine, Hiyosi-cho, Nantan-shi, Kyoto, 629-0392, Japan. Email:

ttaguchi@meiji-u.ac.jp

Received: November 10, 2012

DOI : $10.5430 /$ jnep.v3n10p10
Accepted: December 30, 2012

URL: http://dx.doi.org/10.5430/jnep.v3n10p10

\section{Abstract}

Background: Bright light treatment aims to improve circadian rhythms in patients living indoors with poor light-dark transitions by complementing insufficient natural light and highlighting the difference between their day- and night-time activities. In addition to bright light treatment, it is called "phototherapy" or "high-intensity light therapy", and has been reported to be effective to prevent delirium and sleep disorders in the elderly. Under such circumstances, this study examined the validity of bright light treatment as a perioperative care approach for the elderly who are regarded as vulnerable to delirium due to hospitalization or therapeutic intervention.

Methods: The study was conducted in the mixed surgical ward of approximately 200 beds, located in Kyoto Prefecture. Subjects were elderly male and female patients who underwent surgery for lower limbs, mainly the femur, and were randomly divided into intervention and control groups. The intervention group received bright light treatment with approximately 2,500 lx of bright light from the day after admission; on the day of surgery and at postoperative week 1, while the control group was treated with normal light during this period. The evaluation of delirium was conducted based on the results of nurses' observation and the Japanese-Version NEECHAM Confusion Scale (NEECHAM scale). In addition to this, information regarding the patients' activities of daily living was also collected. As physiological indices, light-exposure-dependent changes in blood serotonin levels and stress-dependent changes in blood cortisol levels were also evaluated. The period of the study was between 2008 and 2010; data were collected from November to February in each year, when the number of target patients increased.

Results: As a result, data regarding 24 patients were obtained within this 2-year period. Excluding those with severe dementia, a total of 16 (intervention: 6; and control: 10) were studied. As a result, delirium occurred in the patients who underwent surgery of the lower limbs, mainly the femur, within the period between the day of surgery and postoperative day 3. The type of delirium was hyperactive in all cases, and its symptoms persisted for 3 to 4 days.

Conclusion: The incidence of delirium was generally lower in the intervention than in the control group, suggesting the validity of bright light treatment.

\section{Key words}

Bright light treatment, Perioperative delirium, Elderly patients 


\section{I ntroduction}

Bright light treatment aims to improve circadian rhythms in patients living in indoor environments with poor light-dark transitions by complementing insufficient natural light and highlighting the difference between their day- and night-time activities. In addition to bright light treatment, it is called "phototherapy” or "high-intensity light therapy”. In the 1980's, light was first identified as the most important factor to synchronize individuals' circadian rhythms with external environments, and has been increasingly focused on for the treatment of sleep disorders since then ${ }^{[1]}$. Particularly, it has been demonstrated that morning light exposure is a strong synchronizing factor playing an important role in improving our circadian rhythms. Further, it has recently been reported that artificial light exposure is effective to treat sleep-wake disorders in dementia and abnormal behavior involved in them, such as delirium and wandering ${ }^{[2-5]}$.

From 2003 to the time of this study, the author examined the association between circadian rhythms and light environments in patients admitted to the intensive care unit (ICU) during the postoperative period. As a result, circadian rhythms tended to be weak or insufficient in patients admitted to the ICU with poor light-dark transitions, showing a poor functional prognosis ${ }^{[6,7]}$. Similarly, patients receiving bright light treatment in the ICU after surgery for esophageal cancershowed a lower incidence of delirium and tendency to recover earlier, compared to those without bright light treatment $^{[8,9]}$.

Based on these findings, this study examined perioperative patients requiring bed rest in terms of delirium prevention. Femoral and other lower-limb fractures frequently occur in the elderly, particularly females with fragile bones due to osteoporosis. Further, 95\% of such fractures occur due to falls in approximately 100,000 individuals every year in Japan; with aging, this number is likely to further increase in the future ${ }^{[10]}$. Delirium tends to develop in a large number of patients with fractures, requiring long-term bed rest in an unfamiliar hospital environment from the preoperative period ${ }^{[11,13]}$. This paper reports its results, while discussing appropriate care approaches based on light-environmental adjustment for perioperative patients.

\section{Background}

\subsection{Hypothesis and objectives}

This study was based on the hypothesis that bright light treatmentis effective to reset circadian rhythms, similarly to morning light exposure, preventing the occurrence of delirium by appropriately adjusting circadian rhythms and highlighting the difference between day- and night-time activities. The evaluation of delirium was conducted based on both nurses' observation and a reliable and valid scale. As postoperative delirium is considered to occur due to mutually associated diverse factors, delirium was examined, not only in terms of its incidence, but also using physiological indices to comprehensively analyze the effect of bright light treatment to appropriately adjust circadian rhythms. The objective of this study was to clarify whether bright light treatment, provided for patients who underwent surgery of the lower limbs, mainly the femur, from the preoperative period to the end of postoperative week 1 , lowers the incidence of deliriumin comparison with those treated without bright light during this period.

\subsection{Characteristics}

This study was characterized by the following 2 points. The first one iscombining the chronobiological and nursing viewpoints, the former of which was taken to examine circadian rhythms corresponding to biological temporal rhythms in living organisms, such as daily, annual, and tidal rhythms. In short, data obtained from patients were chronobiologically analyzed to clarify in-vivo changes as a basis for nursing care. The second is being a cooperative study aiming to examine the challenging topic of the provision of light as a perioperative care approach for the prevention of delirium. 
This study shows originality in being conducted with a perspective on future clinical application, based on the results of previous studies. The novelty of this study is providing patients requiring bed rest with light for therapeutic purposes from the preoperative to the end of the perioperative period. Further, considering daily changes in sunshine conditions, depending on the weather and direction of the bedroom, it aimed to improve circadian rhythms by ensuring a stable sunshine condition with artificial lighting systems.

\subsection{Expected outcomes and significance in terms of nursing care}

The physiological effects of natural or artificial light entering the retina and infiltrating through the optic nerve and nucleus supraopticus include: the adjustment of circadian rhythms; enhancement of arousal levels; adjustment of the autonomic nervous system; and inhibition of melatonin secretion. In line with this, this study examined methods applicable in hospital environments, focusing on the importance of sunlight highlighted by F. Nightingale ${ }^{[14]}$. Further, it aimed to appropriately adjust circadian rhythms by performing bright light treatment from the preoperative to the end of the perioperative period for the first time. The loss of sleep due to hospitalization- or surgery-related anxiety and preoperative rest may be factors contributing to the onset of delirium; therefore, in this study, the possibility of preventing postoperative delirium by appropriately adjusting circadian rhythms from the preoperative period was considered.

The evaluation of daily fluctuations in blood serotonin and cortisol levels to examine the effect of bright light treatment on the adjustment of circadian rhythms may provide a chronobiological viewpoint, simultaneously supporting the validity of such an approach. The provision of improved perioperative light environments may not only prevent delirium, but also enhance nurses' and other health care professionals' awareness of the necessity of providing care in consideration of patients' circadian rhythms.

Bright light treatment for the elderly and patients with severe conditions may also be a drug-independent, non-invasive approach reducing their burden during treatment. If the results of this study are successfully generalized, it may be possible to perform bright light treatment as part of perioperative nursing care appropriate for a rapidly aging society, contributing to the prevention of delirium and shortening of the hospital stay.

\section{Methods}

A pilot study was initially conducted with the approval of the author's university and research ethics committee of the cooperative hospitals, and, subsequently, a full-scale experimental intervention study after the revision and addition of projects.

(1) Design: Experimental intervention (single-blind) study

(2) Patients: Elderly male and female patients undergoing surgery of the lower limbs, mainly the femur, without mental or ophthalmological diseases, or elevated intraocular pressure

(3) Study facility:Themixed surgical ward of a hospital with approximately 200 beds, located in A City, Kyoto Prefecture.

(4) Intervention procedures (see Figure 1)

1) Selected surgical procedures were explained to the patients and their key persons to obtain their written consent.

2) Information regarding the patients' backgrounds was collected from their medical records and through communication with them

3) Bright light treatment was initiated in the morning of the day after admission.

3)-1: The treatment was continuously performed until the end of postoperative week 1 , including on the day of surgery. 
3)-2: Based on the findings from preceding studies and specialists' opinions, the patients were exposed to the light source placed at the foot or on the left or right side of the bed for 2 hours from 7:00 to 9:00 at a distance (in the preocular horizontal plane) of approximately $1 \mathrm{~m}$ (see Figure 2).

3)-3: As the light source, a box-type lighting device (Bright Light ME; Over The Top Inc.), approved for medical purposes, was used. The level of bright light irradiated on the patients' faces was set at approximately 2,500 lx with a view to effectively obtain the therapeutic effect.

4) The presence/absence of delirium was evaluated during 7-day pre- and postoperative periods based on nurses' observation and the delirium scale.

5) To evaluate the effect of bright light treatment on the adjustment of circadian rhythms during the pre- and postoperative periods, blood serotonin and cortisol levels were quantified. Blood samples were collected in the morning on the day before surgery, and on postoperative days 1,3,5, and 7, conforming to the doctor's instructions.

6) The patients' conditions were evaluated by collecting information from nurses to examine their timedependent changes.

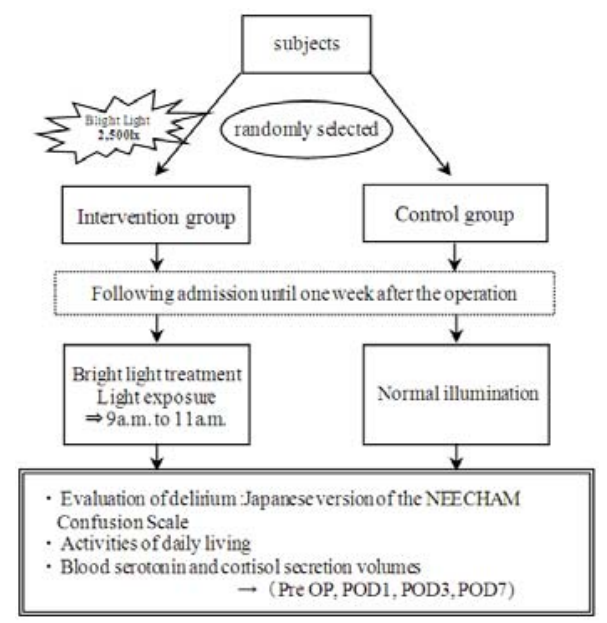

Figure 1. Procedure of Bright light treatment

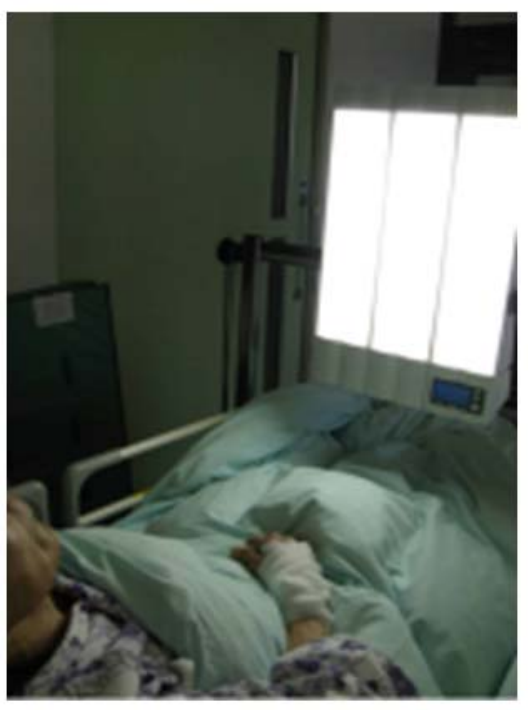

Figure 2. Bringt light treatment for patients 


\section{Analysis}

The association between bright light treatment, the presence/absence of delirium, and blood serotonin/cortisol levels was analyzed by performing Pearson'schi-square, Friedman's nonparametric, and Mann-Whitney tests. The significance level was set at $5 \%$ or less of the risk rate on both sides.

\section{Period of the study}

This study was conducted within the period between 2008 and 2010; from November to February in each year as a period when the number of target patients and surgery for them was the greatest.

\section{Ethical considerations}

Some preceding studies have reported that no side effects were observed in hospitalized patients treated with higher bright light levels, using artificial lighting devices ${ }^{[2,8,9,15]}$. However, it may be necessary to consider the possibility for such an intervention approach to light environments as a physical environmental factor to cause stress in perioperative patients who tend to be mentally and physically unstable. Based on this idea, the present study was conducted with the approval of the author's university and research ethics committee of the cooperative hospitals, as well as the patients' written consent. The patients were previously provided with an explanation regarding the absolute right to withdraw from the study after consenting to it. Ethical considerations also included privacy protection and the publication of results for academic meetings and journals, avoiding the identification of individuals. The collected data were carefully managed and protected by the researchers, using computers. This study does not create conflicts of interest with any company.

\section{Results}

A total of 16 patients, 2 males and 14 females, were studied (see Table 1), and randomly divided into intervention (6) and control (10) groups.

While data regarding 24 patients were initially obtained, a total of 16 meeting the above-mentioned requirements, excluding those with severe dementia, were studiedand classified into intervention and control groups based on the enveloped method. Table 1 shows their clinical histories; all of them had undergone oral treatment for previous diseases. The mean age and standard deviation were $85.1 \pm 7.1$ in the former, and $84.6 \pm 6.1$ in the latter group; there were no significant differences in the age, duration of surgery, and amount of blood loss between them. In both groups, all patients, except for one, were treated with extradural anesthesia for postoperative pain control. The patients were admitted to bedrooms for 2 or 4 persons to undergo surgery due to fractures of their lower limbs, mainly femora. The level of brightness around the center of their bedrooms was 500 to $600 \mathrm{~lx}$ during the day-time. The lights were turned off at 21:00 at night, and turned on again after 6:00 in the morning.

In the intervention group, bright light treatment was initiated in the morning of the day after admission by irradiating the patients' face with approximately 2,500-lx light with the lighting device placed at the foot or on the left or right side of the bed for 2 hours from 7:00 to 9:00. As the position of the bed varied among patients—some faced the window, and others the corridor, the level of brightness was measured and adjusted per situation. The treatment was continuously performed until the end of postoperative week 1.The mean duration of the treatment provided from immediately after admission, including on the day of surgery, was 1.6 days.

\section{1) Incidence of delirium and its patterns}

The incidence of delirium was $0 \%$ ( 0 of 6$)$ in the intervention and $40 \%$ (4 of 10$)$ in the control groups. The result of Pearson's chi-square test was $P=0.076$; the intervention group showed a slightly lower incidence. On comparison using the delirium scale, there was a tendency for both groups to show a low and fluctuating incidence of delirium within the period 
from surgery to postoperative day 3. Further, delirium scores increased after postoperative day 4 in all 6 patients of the intervention group. Such an increase was also observed in 7 of 10 in the control group. Regarding periods for the onset of delirium, it occurred at night on the day of surgery in 1, on postoperative day 1 in 2 , and on postoperative day 3 in 1 . All of them presented with symptoms of hyperactive delirium. Among the patients with delirium in the control group, as shown in Table 1, nocturnal awakenings and dangerous behavior were observed from the day of surgery until postoperative day 4in patient K. Similarly, in patient $\mathrm{H}$, delirium occurred on postoperative day 1 , and impaired orientation was observed until postoperative day 2. Delirium also occurred on postoperative day 1in patient J, involving marked symptoms of hyperactive delirium until postoperative day 4 , such as screaming and removing the bed-rail and infusion tube. In patient G, dangerous behavior, such as standing on the bed, was observed, in addition to hallucinatory images, such as worms in the ceiling.

Table 1-1. Back ground of patients (Intervention Group)

\begin{tabular}{|c|c|c|c|c|c|c|c|c|c|}
\hline No & Sex & Age & Diagnosis & Medical History & Operation & Delirium & $\begin{array}{l}\text { Op } \\
\text { time(min) }\end{array}$ & $\begin{array}{l}\text { blood } \\
\operatorname{loss}(\mathrm{ml})\end{array}$ & $\begin{array}{l}\text { Epidural } \\
\text { anesthesia* }\end{array}$ \\
\hline $\mathrm{A}$ & $\mathrm{F}$ & 86 & Right femoral neck fracture & Heart failure & R UHA & None & 110 & 300 & $\mathrm{O}$ \\
\hline B & $\mathrm{F}$ & 86 & Right trochanteric fracture & None & RITST & None & 43 & 50 & O \\
\hline $\mathrm{C}$ & $\mathrm{F}$ & 87 & $\begin{array}{l}\text { Right supracondylar fracture of } \\
\text { the femu }\end{array}$ & None & L Unreamed nail & None & 85 & 100 & $x$ \\
\hline $\mathrm{D}$ & $\mathrm{F}$ & 77 & Right femoral neck fracture & Angina & Hanson-pin & None & 39 & 30 & O \\
\hline $\mathrm{E}$ & $\mathrm{F}$ & 102 & Left trochanteric fracture & $\begin{array}{l}\text { Htoertension, } \\
\text { arrhythmia }\end{array}$ & OFIA (CHS) & None & 86 & 350 & O \\
\hline $\mathrm{F}$ & $\mathrm{M}$ & 86 & Left thigh bone myelitis & Cerebral infarction & $\begin{array}{l}\text { Foreign body } \\
\text { removal }\end{array}$ & None & 30 & $\begin{array}{l}\text { A small } \\
\text { quantity }\end{array}$ & O \\
\hline
\end{tabular}

*Epidural Anesthesia: for post-operative pain

Table 1-2. Back ground of patients (Control Group)

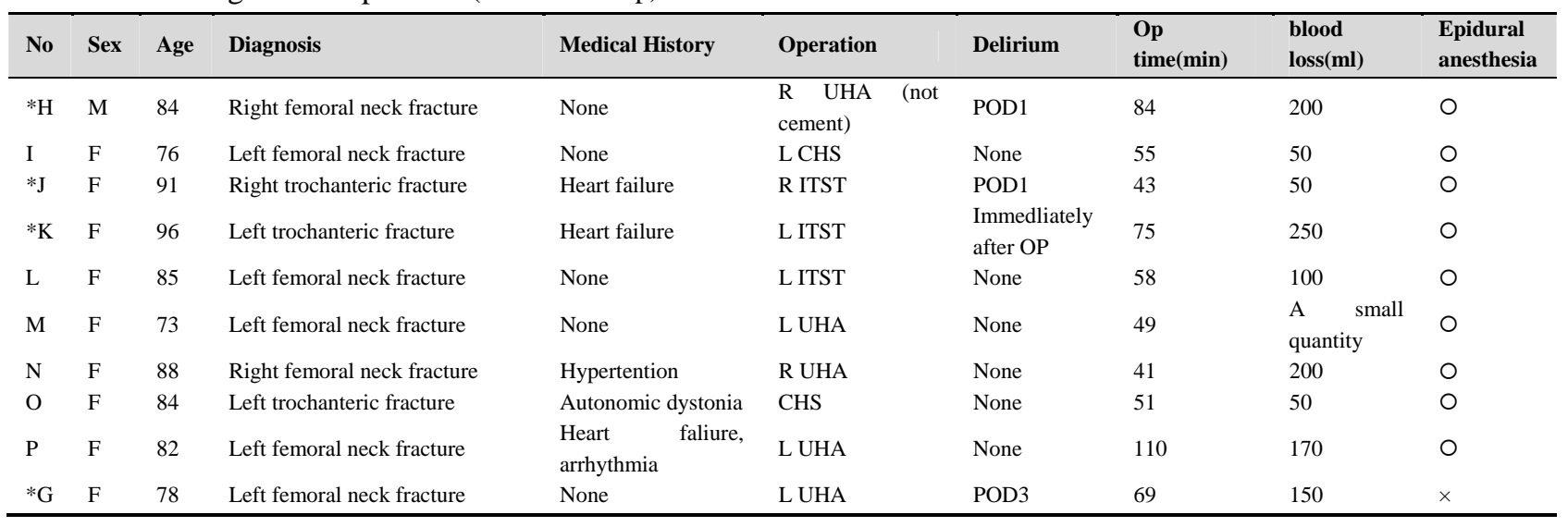

*H, J, K, G : Delirium patients

\section{2) Patients' responses to bright light treatment}

Although detailed interviews with the 6 patients treated with bright light treatment were not conducted, the majority of them stated that they had a relatively favorable feeling on rising after treatment. On the other hand, none of them had high-quality sleep underthe presence of surgical wound and lower-back pain due to postoperative rest care. While there was no withdrawal from the treatment, 1 patient stated, "I feel a little dazzled and hot" with it. No side effects due to bright light exposure were observed in any of the intervention group.

\section{3) Analysis based on physiological indices}

Significant differences in physiological indices were examined by performing Friedman's test. No significant increase in the blood serotonin level was observed during the postoperative period in either group. In contrast, the blood cortisol level 
significantly decreased in the control group, although such changes in blood serotonin and cortisol levels markedly varied among individuals, depending on their personal patterns.

\section{Discussion}

In this study, patients undergoing surgery of the lower limbs, mainly the femur, were studied in comparison between with and without bright light treatment from the preoperative period. As a result, it was demonstrated that the incidence of delirium was $0 \%$ in the former group; however, on statistical analysis, this result was solely shown as a tendency. As no significant increase in blood serotonin levels was observed even in the intervention group, evidence for bright light treatment was not clearly obtained in this study. In line with this, further studies may be necessary to examine evaluation indices in order to sufficiently consider differences among patients undergoing surgery. In the present study, blood serotonin and cortisol levels were considered as light-exposure- and stress-dependent indices, respectively; however, considering that these hormones in the blood were likely to be influenced by anesthesia and surgical invasion, it may be more appropriate to use blood melatonin levels in order to evaluate the direct effects of bright light treatment. Further, in almost all patients, postoperative pain persisted despite continuous treatment with extradural anesthesia for pain control; therefore, it may also be necessary to consider the presence of pain as an additional index.

Kuroiwa et al. examined the incidence of delirium in 32 patients aged 65 and over, who were emergently hospitalized due to femoral head fractures, and treated with femoral head replacement, reporting that delirium scores decreased during the pre- and postoperative periods, and increased on postoperative day 3 in $80 \%$ of these patients. In the present study, delirium scores tended to be low and fluctuating from the day of surgery until postoperative day 3 in both groups. Further, the scores increased after postoperative day 4 in all patients of the intervention and 7 of the control group, corresponding to the most frequent period for the onset of postoperative delirium ${ }^{[11]}$.

Similarly, in a study conducted by Miyazaki et al. to examine factors contributing to the incidence of postoperative delirium in 161 patients who underwent joint replacement surgery and 29 who underwent knee joint replacement, the incidence of delirium was $11 \%$; it occurred most frequently on the day of surgery and postoperative day 1 , with a mean duration of 2 days. In the present study, delirium occurred on postoperative day 1 in 2, showing a similar result, while persisting for a longer period of time ${ }^{[12]}$. Based on the results of the preceding and present studies, the elderly undergoing surgery of the lower limbs, mainly the femur, may be vulnerable to the onset of delirium from the preoperative period; particularly, their conditions may be markedly unstable until 3 postoperative day. Therefore, it may be important to initiate delirium-preventing approaches during the preoperative period, and chronological evaluation may play an essential role in this.

In the course of normal life, it is possible for all of us to unexpectedly fall ill and need hospitalization ${ }^{[16]}$. On the other hand, with the recent progress in medical treatment, opportunities to receive surgery are open even to the elderly. It is difficult for perioperative patients requiring bed rest to improve their living rhythms by themselves. They have no choice but to live in an environment giving priority to treatment. Under such circumstances, this study examined the most effective method to recover patients' natural circadian rhythms in consideration of the necessity of artificially controlling their light environments, focusing on the importance of sunlight highlighted by F. Nightingale. Marked progress has been made in modern medicine since the discovery of anesthesia, such as the development of innovative surgical procedures and cutting-edge treatment methods ${ }^{[16]}$. On the other hand, it is true that hospitals have developed as a system centering on health care professionals, rather than patients. Although the importance of providing health care and nursing services from the patient's standpoint has been increasingly focused on in recent years, it is still questionable whether they are actually provided, while fully considering individuals' characteristics.

Unlike doctors performing treatment, nurses are in charge of 24-hour bedside patient monitoring; therefore, it is necessary for them to understand the characteristics of patients' circadian rhythms based on their life activities prior to 
hospitalization in order to provide nursing care appropriate for each individual. While the patients were treated with artificial morning light in this study, it may also be possible to provide such intervention approaches using natural light by enhancing nurses' understanding of the biological mechanisms of circadian rhythm adjustment.It has been reported that a sleep-promoting hormone, called melatonin, is appropriately secreted 14 to 15 hours after sufficient exposure to morning light ${ }^{[17]}$. In contrast, exposure to bright light after late afternoon suppresses the secretion of melatonin, leading to a delayed circadian rhythm ${ }^{[18]}$. In line with this, it may be appropriate for nurses to deepen their knowledge regarding such effects of light, while accurately understanding individual patients' light environments in their bedrooms using an illuminometer. Based on the findings from these approaches, it may be necessary for them to review their daily nursing care, and devise more effective approaches, such as increasing the amount of natural light entering patients' bedrooms and changing the time of their outdoor walking. Further, on the extension and structural alteration of the hospital or bedrooms, it may be important to reconsider various factors, such as the direction and size of the windows, and the possibility of installing a space for sun-bathing.

The human body maintains health by protecting its stable internal environment from diverse external stimuli, such as changes in the temperature, humidity, atmospheric pressure, and light, electrical and mechanical stimulation, and mental stress. Such natural healing power is called spontaneous cure ${ }^{[19]}$. Spontaneous cure generally means the congenitally acquired ability of humans and animals to cure their own mental and physical injuries and diseases; in other words, it is an ability to cure without the administration of artificial agents. Ho developed a lighting system enabling residents of long-term care health facilities to bathe in natural light while watching TV in the day-time activity room, and reported that it was effective to promote their sleep ${ }^{[20]}$. Further, in Dr. Mariah Snyder’s Complementary \& Alternative Therapies in Nursing, Sixth Edition, light is regarded as a complementary alternative therapy approach, suggesting the necessity of performing such an approach in nursing practice ${ }^{[21]}$. The adjustment of circadian rhythms based on bright light treatment may be a drug-independent, non-invasive approach improving the ability of spontaneous cure by normalizing patients' circadian rhythms and promoting their immune and self-renewal systems. An improved functional prognosis may alleviate patients' mental and physical distress from the early stages, while reducing their financial burden, and increasing their QOL.

\section{Limitations and future perspectives}

It is difficult to generalize the results of this study due to the small number of patients. Further, the results of physiological evaluation varied among individuals; it was also difficult to conduct such an evaluation for a long period of time, using indices requiring blood sample collection. On the other hand, in order to conduct such an experimental intervention study over a long period of time, it is necessary to obtain cooperation from clinical staff by systematically organizing a project team consisting of researchers, clinical staff, and specialists, in which they mutually cooperate.

\section{Conclusion}

On comparison between the intervention and control groups who underwent surgery of the lower limbs, mainly the femur, the incidence of delirium was as low as $0 \%$ in the former after bright light treatment provided from the preoperative period to the end of postoperative week 1.

\section{Acknowledgement}

The author would like to thank all patients and hospital staff members who cooperated with this study over a long period of time. This study was conducted as challenging exploratory research with a Grant-in-Aid for Scientific Research 2009 to 2011. 


\section{References}

[1] Koyama E, Matsubara H, Nakano T. Bright light treatment for sleep-wake disturbances in aged individuals with dementia.

Psychiatry and Clinical Neurosciences. 1999; 53(2):227-229. PMid:10459695

http://dx.doi.org/10.1046/j.1440-1819.1999.00483.x

[2] Kobayashi R, Fukida N, Kohsaka M, et al. Effect of bright light at lunchtime on sleep of patients in a geriatric hospital I .

Psychiatry and Clinical Neurosciences. 2001; 55(3):287-289. PMid:11422879

http://dx.doi.org/10.1046/j.1440-1819.2001.00863.x

[3] Hanford N, Figueiro M. Light Therapy and Alzheimer's disease and Related Dementia: Past, Present, and Future. J. Alzheimer's Dis. 2012 Oct.; 25: 1-10.

[4] Yang J, Choi W, Ko YH et al. Bright light therapy as an adjunctive treatment with risperidone in patients with delirium. A randomized, open, parallel group study. Gen Hosp Psychiatry. 2012; 34(5): 546-551. PMid:22717090 http://dx.doi.org/10.1016/j.genhosppsych.2012.05.003

[5] Takahashi K. 2011: Handbook of sleep science and sleep medicine, 4th edition, The Japanese Society of Sleep Research, Tokyo.

[6] Kropyvnytsky I, Saunders F., Pols M.et al. Circadian rhythms of temperature in head injury. Brain Inj. 2001; 15(6):511-518. PMid:11394970 http://dx.doi.org/10.1080/02699050010007515

[7] Sumaya IC, Rienzi BM, Deegan JF et al. Bright Light Treatment Decreases Depression in Institutionalized Older Adults; a Placebo-Controlled Crossover Study. Journal of Gerontology. 2001; 56(6):356-360.

[8] Taguchi T, Yano M, Kido Y. Influence of bright light therapy on postoperative patients. A pilot study. Intensive and Critical Care Nursing. 2007; 23(5): 289-297. PMid:17692522 http://dx.doi.org/10.1016/j.iccn.2007.04.004

[9] Ono H, Taguchi T, Kido Y. et al. The usefulness of bright light therapy for patients after oesophagectomy. Intensive and Critical Care Nursing. 2011; 27 (3):158-166. PMid:21511473 http://dx.doi.org/10.1016/j.iccn.2011.03.003

[10] Kato K. (2012): System science of nursing 10 Orthopedics, Igakushoin Publishing Co., Ltd. Tokyo.

[11] Miyazaki K, Tanaka K, Tomizawa C. et al. A Study on Factors Associated with the Incidence of Delirium in Patients Undergoing Joint Replacement Surgery. Orthopedic Surgical Nursing Care. 2008; 13(4):418-423.

[12] Kuroiwa M, Matsuoka M, Kondo S. Postoperative Delirium of Patients Who Underwent Femoral Head Replacement. Hip Joint. 2007; 3: 25-27.

[13] Goldenberg Gregory, Kiselev Pavel, Bharathan Thayylathil et al. Predicting postoperative delirium in elderly patients undergoing surgery for hip fracture. 2006; 6(2):43-48.

[14] Usui H, Konami Y. 1860: Notes on nursing. Gendaisha Publishing Co. Ltd, Tokyo.

[15] Gallin P. F, Terman M, Reme C E. Ophthalmologic Examination of patients with seasonal Affective Disorder, Before and after Bright Light Therapy. American Journal of Ophthalmology.1995; 119(2): 202-210.

[16] Hattori S.(2004):The Light and Shade of Modern Medicine, Yamakawa Shuppansha, Ltd. Tokyo.

[17] Miyazaki S, Sato N, Ohkawa M. et al. (2011): Sleep study introduction handbook, Japanese sleep machinery of education. Tokyo.

[18] Sato A, Rishiri T, Ibuka N.et al. (1997): Handbook of Human and Light, 2nd edition. Asakura Shoten, Tokyo.

[19] Yano T, Guide to Oriental Medicine (1998): NicchuShuppan, Co.,Ltd. Tokyo.

[20] Ho SC, Wong T, KS, Tang PL et al. Nonpharmacologic sleep promotion;bright light exposure. Complementary Therapies in Nursing \& Midwifery. 2002; 8(3):130-135. http://dx.doi.org/10.1054/ctnm.2002.0630

[21] Mariah Snyder: Complementary \& Alternative Therapies in Nursing 6th edition, Springer publishing Company, New York, 2009. 